\section{How microsatellite analysis can be exploited for subtelomeric chromosomal rearrangement analysis in mental retardation}

EDITOR - The genetic causes of mental retardation are still largely unknown so that about $34 \%$ of cases of severe to moderate and $80 \%$ of mild mental retardation remain unresolved. ${ }^{12}$ Consequently, genetic counselling is difficult in these cases. Chromosomal rearrangements are still the most frequent cause of mental retardation and cytogenetic analysis at 400-550 band resolution cannot detect rearrangements smaller than $5 \mathrm{Mb}$. Therefore, any new technologies to improve cytogenetic analysis would be of great benefit. In recent years, there has been evidence that the cause of $6-7 \%$ of mental retardation involves subtle chromosome rearrangements.

Several molecular methodologies have been used successfully to investigate the integrity of telomeres, such as hypervariable polymorphisms (HVPs), FISH, and CGH, while others based on DNA array approaches are being developed. ${ }^{2}$ The choice of method rests on considerations of feasibility, cost, reproducibility, and sensitivity. FISH and microsatellite analyses are the techniques most commonly used by numerous laboratories and both of them are still being evaluated in terms of their performance and practicability. FISH based analysis with telomeric specific probes provides a simultaneous investigation of all chromosomes ends, resulting in total detection of chromosome rearrangements without requiring the examination of parental chromosomes. ${ }^{3-5}$ However, this method does not assign parental origin, it loses UPD events, and only gives information regarding abnormalities spanning the probe's DNA region. Although now simplified by being available in kit form, the method not only requires considerable expertise in molecular cytogenetics, but is also expensive and time consuming.

HVP analysis using microsatellites, which is both easy and inexpensive, has two main problems, as it requires both parental DNA samples and high informativeness, established by the number and frequencies of alleles. However, even for highly heterozygous loci $\cong 0.8$, the detection rate for monosomy and trisomy is 0.64 and 0.5 , resulting in about $50 \%$ of potential carriers for subtelomeric rearrangements remaining undetected. ${ }^{6}$

In order to underline how microsatellite analysis can give a detection rate matching that obtained using FISH, we performed a comparative pilot investigation on 60 unrelated mentally retarded patients using a collection of microsatellite polymorphisms mapping chromosomal subtelomeric regions and telomere specific probes according to the multiprobe FISH protocol, as previously described by Knight et al. ${ }^{3}$

From the 60 subjects selected for the study, 30 (group 1) were already part of a larger FISH based screening for cryptic telomeric rearrangements on 200 Italian mentally retarded patients. ${ }^{7}$ The remaining 30 subjects (group 2) were referred by clinical geneticists because their clinical characteristics strongly suggested chromosomal abnormalities. Fundamental clinical criteria for both groups included: (1) a degree of mental retardation ranging from moderate (IQ 50 to 70 ) to severe (IQ <50), (2) a normal karyotype, (3) absence of diagnosis, and (4) no known consanguinity. Multiple congenital anomalies (MCA) and dysmorphism were present in 40 of the 60 subjects included in the study. FISH analysis with telomere specific probes (Cytocell multiprobe kit) was performed on fixed lymphocytes metaphase chromosomes, prepared directly from peripheral blood, as previously described. ${ }^{3}$ A total of four subtle telomeric rearrangements were found by this method in 60 subjects. Two de novo deletions (46,XY,6qand $46, \mathrm{XX}, 2 \mathrm{q}-$ ) carried by two probands in group 1 have been described elsewhere. ${ }^{7}$ In subjects in group 2, FISH analysis showed two different unbalanced derivatives: $46, X Y, \operatorname{der}(15 p) t(15 p ; 17 p)$ and $46, X X, \operatorname{der}(4 p) t(4 p ; 8 p)$. The clinical presentations will be reported elsewhere. The unbalanced derivatives $4 p ; 8 p$ and $15 p ; 17 p$ were easily shown by FISH in the probands as well as the balanced translocation in the carrier mothers. For the translocation $15 \mathrm{p} ; 17 \mathrm{p}$, the rearrangement was confirmed by FISH using specific YAC probes obtained from the Screening YAC Center, since the kit used does not include probes for the $p$ arms of acrocentric chromosomes.

The percentage of anomalies disclosed in our sample by this method was $6.6 \%$, matching the frequency obtained in others studies. ${ }^{4}$ Since all subjects from both groups were studied simultaneously, no bias is represented by the group 1 subjects being involved in another study. For HVP analysis using microsatellites, we initially designed a panel of 41 subtelomeric markers (table 1) chosen on the basis of their informativeness (Het range 0.52-0.93) and telomeric position. Twenty five of the markers had a physical distance of 0-2 Mb from the chromosomal ends. The remainder were genetically mapped and represent those most telomeric and informative. Their distance from the telomere was assigned according to the integrated mapping resource (www.gdb.org). The genetic distribution was spread over a range of $<2.5 \mathrm{cM}$.

Manual genotyping was performed with multiplex PCR on 96 well polycarbonate plates, amplifying three distinct polymorphic loci per single reaction mix simultaneously. For each family trio (proband and parents), 41 PCR were performed, that is, 13.6 per person. It should be noted that for complete use of the microsatellite panel only about $2 \mu \mathrm{g}$ of DNA is necessary per sample. Detection of allelic segregation pattern was achieved by two alternative methods, the first was that described by Dib et al and the second used the ABI-PRISM 302 (Perkin-Elmer) microsatellite analyser, as recommended by the manufacturer. Detailed protocols are available from $\operatorname{Dr} M$ Fichera (mfichera@oasi.en.it).

In our sample, microsatellite analysis showed the monosomy $2 \mathrm{q}$ and $6 \mathrm{q}$ in group 1 . The derivatives $4 \mathrm{p}-$ and $15 \mathrm{p}-$ from subjects with unbalanced translocations (group 2) were not detected.

In 60 probands analysed by this method, given that the detection rate (DR) for monosomy of our panel is 0.59 , the expected number of monosomic cases would be 3.4. compared to the four cases disclosed by FISH. Both trisomies from the group 2 patients were missed.

The limitations of a microsatellite panel including only one marker for the telomere prompted us to construct an enriched panel of three polymorphic markers spread over a defined telomeric DNA region. The aim of using three 
Table 1 Proposed panel of 41 microsatellites markers

\begin{tabular}{|c|c|c|c|c|c|}
\hline Chr & Marker name & $\begin{array}{l}\text { Distance from } \\
\text { telomere }(\mathrm{kb})\end{array}$ & Het $\mathrm{O}$ & $I M O$ & ITO \\
\hline $1 \mathrm{p}$ & $\mathrm{D} 1 \mathrm{~S} 243$ & & 0.86 & 0.68 & 0.58 \\
\hline $1 \mathrm{q}$ & D1S3739 & $80^{\star}$ & 0.66 & 0.49 & 0.28 \\
\hline $2 p$ & $2 p$ & $<501 \dagger$ & 0.84 & 0.63 & 0.53 \\
\hline $2 q$ & D2S2986 & $240^{\star}$ & 0.57 & 0.42 & 0.23 \\
\hline $3 p$ & D3S4559 & $450^{\star}$ & 0.66 & 0.53 & 0.31 \\
\hline $3 q$ & D3S4560 & $450^{\star}$ & 0.80 & 0.61 & 0.49 \\
\hline $4 \mathrm{p}$ & D4S3359 & $86.5+$ & 0.57 & 0.48 & 0.25 \\
\hline $4 q$ & D4S2930 & $300-700^{\star}$ & 0.75 & 0.53 & 0.39 \\
\hline $5 p$ & D5S2005 & & 0.74 & 0.45 & 0.30 \\
\hline $5 q$ & D5S2006 & $30 \ddagger$ & 0.80 & 0.50 & 0.40 \\
\hline $6 \mathrm{p}$ & D6S1600 & & 0.72 & 0.66 & 0.43 \\
\hline $6 q$ & 6 qtel 53 & $262 t$ & 0.84 & 0.66 & 0.55 \\
\hline $7 \mathrm{p}$ & D7S2477 & $0 \ddagger$ & 0.74 & 0.48 & 0.33 \\
\hline $7 \mathrm{q}$ & $7 \mathrm{q}$ & $7 \dagger$ & 0.76 & 0.70 & 0.50 \\
\hline $8 \mathrm{p}$ & D8S504 & $250-450^{\star}$ & 0.79 & 0.52 & 0.35 \\
\hline $8 \mathrm{q}$ & D8S2334 & & 0.88 & 0.64 & 0.56 \\
\hline $9 \mathrm{p}$ & D9S 1858 & & 0.63 & 0.37 & 0.23 \\
\hline $9 q$ & D9S1838 & $300 \ddagger$ & 0.87 & 0.77 & 0.65 \\
\hline $10 \mathrm{p}$ & 10 ptel35 & $0 \ddagger$ & 0.84 & 0.63 & 0.53 \\
\hline $10 \mathrm{q}$ & 10qtel 23 & & 0.52 & 0.36 & 0.22 \\
\hline $11 \mathrm{p}$ & 11 ptel03 & $125^{\star}$ & 0.90 & 0.78 & 0.70 \\
\hline $11 \mathrm{q}$ & D11S4112 & & 0.75 & 0.43 & 0.28 \\
\hline $12 p$ & D12S352 & & 0.76 & 0.49 & 0.32 \\
\hline $12 \mathrm{q}$ & 12 qtel04 & & 0.87 & 0.72 & 0.61 \\
\hline $13 \mathrm{q}$ & 13 qtel10 & & 0.61 & 0.41 & 0.19 \\
\hline $14 \mathrm{q}$ & 14qtel 04 & $0 \ddagger$ & 0.77 & 0.58 & 0.42 \\
\hline $15 q$ & D15S642 & & 0.81 & 0.70 & 0.56 \\
\hline $16 \mathrm{p}$ & D16S3401 & & 0.80 & 0.74 & 0.57 \\
\hline $16 \mathrm{q}$ & D16S671 & & 0.85 & 0.71 & 0.59 \\
\hline $17 \mathrm{p}$ & D17S1866 & & 0.84 & 0.78 & 0.65 \\
\hline $17 \mathrm{q}$ & D17S928 & & 0.83 & 0.68 & 0.65 \\
\hline $18 \mathrm{p}$ & $18 \mathrm{p}$ & $0 \ddagger$ & 0.75 & 0.73 & 0.50 \\
\hline $18 \mathrm{q}$ & 18qtel 69 & $<321 \dagger$ & 0.89 & 0.74 & 0.67 \\
\hline $19 p$ & D19S886 & & 0.73 & 0.46 & 0.32 \\
\hline $19 q$ & D19S218 & $150 \ddagger$ & 0.67 & 0.40 & 0.19 \\
\hline $20 p$ & D20S1155 & $0 \ddagger$ & 0.83 & 0.72 & 0.57 \\
\hline $20 \mathrm{q}$ & D20S64 & $1990 \ddagger$ & 0.93 & 0.78 & 0.73 \\
\hline $21 \mathrm{q}$ & 21qtel 07 & $175^{\star}$ & 0.81 & 0.59 & 0.48 \\
\hline $22 \mathrm{q}$ & $22 \mathrm{q}$ & $1500 \dagger$ & 0.74 & 0.53 & 0.35 \\
\hline $\mathrm{Xp}$ & DXYS233 & $0 \ddagger$ & 0.83 & 0.64 & 0.52 \\
\hline $\mathrm{Xq}$ & Xqyq & $0 \ddagger$ & 0.70 & 0.60 & 0.41 \\
\hline \multicolumn{3}{|c|}{ Average } & 0.77 & 0.59 & 0.45 \\
\hline
\end{tabular}

${ }^{\star}$ Knight et al. ${ }^{10}$

†GenBank mapped.

$\ddagger$ Slavotinek et al. ${ }^{9}$

Het O: observed heterozygosity. IMO and ITO: observed informativeness respectively for monosomy and trisomy.

consecutive markers was twofold: (1) to increase the informativeness for monosomy and trisomy and (2) to avoid using parental DNA. A pilot model was performed for four telomeres, $2 \mathrm{p}, 3 \mathrm{q}, 18 \mathrm{q}$, and $22 \mathrm{q}$, chosen according to the clone sequences available on the GenBank Data Base. Since $4 \mathrm{p}$ and $8 \mathrm{q}$ are not present in the data bank, these were not selected. The three polymorphic loci in the four telomeres chosen are situated at a mean distance of $0.6 \mathrm{Mb}$ from the telomere. Nine of these 12 microsatellites have already been physically mapped in GenBank. The remaining three $(\mathrm{CA}) \mathrm{n}$, two for telomere $22 \mathrm{q}$ and one for telomere $18 \mathrm{q}$, were selected from the sequences available in the GenBank. The criterion for choosing them was the length of the repeat. The heterozygosity value was verified in our patients and was equal to 0.76 .

Microsatellite analysis using three consecutive polymorphic loci was evaluated for the proband both with and without parental allelic segregation. The results obtained for the four loci are shown in fig 1. The informativeness was calculated for both anomalies, on the principle that at least one of the three loci was informative, $I=1-\left[\left(1-i_{a}\right)\left(1-i_{b}\right)\left(1-i_{c}\right)\right]$ where $i_{n}$ represents the informativeness of each locus. In this way, the monosomy informativeness rises to 0.93 (compared to 0.60 from the analysis of a single locus) and that of trisomy to 0.83 (doubling the DR obtained with a single locus).

Analysing the sample without parental allelic segregation, the result for monosomy is interpreted as the exclusion of the anomaly. The probability of exclusion is given by the presence of at least one of the three loci in heterozygosity,

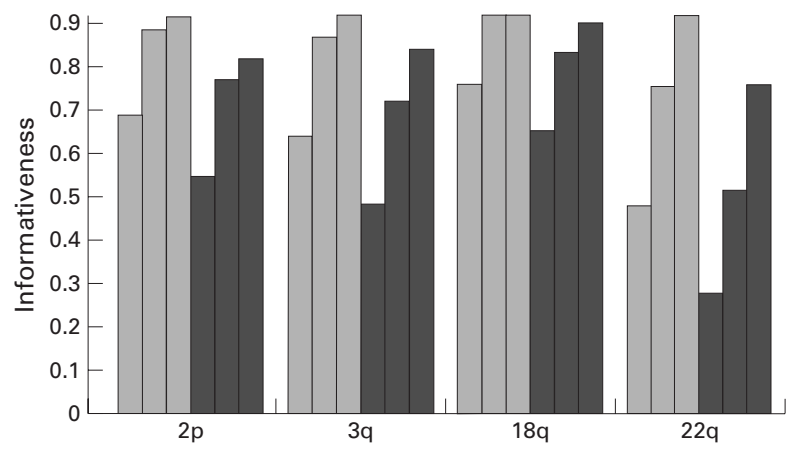

Figure 1 Graphic representation of informativeness for monosomy (grey) and trisomy (black) considering the parental allelic segregation for one, two, and three microsatellites per telomere. New mapped polymorphic markers for telomeres $2 p$ and $22 q$ are located within $700 \mathrm{~kb}$ from their telomere.

therefore $e=1-\left[\left(1-e_{a}\right)\left(1-e_{b}\right)\left(1-e_{c}\right)\right]$ where $e_{n}$ is the heterozygosity of a single locus. Without parental allelic segregation, it is not possible to determine the DR, which depends on the approach chosen to investigate the unresolved cases. Conversely, the DR for trisomy remains the same with or without parental allelic segregation because the presence of three different alleles is obligatory.

No linkage disequilibrium was observed during allelic segregation, guaranteeing the mutual independence of the loci. Nevertheless, if this approach were extended to all the telomeres and linkage disequilibrium were found between three loci, it would be enough to calculate the exclusion of monosomy and the informativeness of trisomy, taking into account the haplotype frequency in the population. In this case both values should be decreased.

This study finally clarifies the limits of resolution of the HVP approach, even when using three aligned loci. Further, an analysis extended to the parents is evidently not necessary because, even if it is impossible to affirm the detection rate for monosomy without the parents, the probability of excluding the anomaly is as high as $98.5 \%$, reducing the number of telomeres to be investigated to $1.5 \%$. For trisomy, however, the missed DR remains $17 \%$.

Even though FISH analysis has a $100 \%$ DR, the approach suggested here represents an easy and economical method which gives acceptable results and, in any case, directs the investigation towards a more focused FISH analysis.

In conclusion, considering that all genomic sequences will soon be deposited in GenBank, this could represent a refinement of the whole genome microsatellite screening recently described by Rosenberg et al. ${ }^{10}$

The first two authors contributed equally to this work. This work was supported by grants ICS.1,1/RF98/81, and RF95.240 from the Italian Health Ministry.

$$
\begin{array}{r}
\text { EUGENIA BORGIONE } \\
\text { MARIANGELA LO GIUDICE } \\
\text { ORNELLA GALESI* }^{\star} \\
\text { LUCIA CASTIGLIA }^{\star} \\
\text { PINELLA FAILLA } \\
\text { CORRADO ROMANO } \\
\text { ANGELA RAGUSA } \\
\text { MARCO FICHERA }
\end{array}
$$

*Laboratorio di Patologia Genetica, IRCCS Oasi Maria SS, Via Conte Ruggero 73, 94018 Troina (Enna), Italy †Unità Operativa di Pediatria, IRCCS Oasi Maria SS, Troina, Italy

Correspondence to: Dr Fichera, mfichera@oasi.en.it

1 Curry CJ, Stevenson RE, Aughton D, et al. Evaluation of mental retardation: recommendations of a consensus conference. Am 7 Med Genet 1997;72:468-77.

2 Knight SJL, Flint J. Perfect endings: a review of subtelomeric probes and their use in clinical diagnosis. F Med Genet 2000;37:401-9.

3 Knight SJL, Horsley SW, Regan R, et al. Development and clinical application of an innovative fluorescent in situ hybridization technique which tion of an innovative fluorescent in situ hybridization technique which
detects submicroscopic rearrangements involving telomeres. Eur f Hum detects submicro

4 Knight SJL, Regan R, Nicod A, et al. Subtle chromosomal rearrangements in children with unexplained mental retardation. Lancet 1999;354:1676-8. 
5 Flint J, Wilkie AOM, Buckle VJ, et al. The detection of subtelomeric chromosomal rearrangements in idiopathic mental retardation. Nat Genet 1995;9:132-9.

6 Wilkie AOM. Detection of cryptic chromosomal abnormalities in unexplained mental retardation: a general strategy using hypervariable subtelomeric DNA polymorphisms. Am f Hum Genet 1993;53:688-701.

7 Rossi E, Ragusa A, Mauro P, et al Cryptic telomeric rearrangements in sub-

jects with chromosomal phenotype and mental retardation. Submitted.
8 Dib C, Faure S, Fizames C, et al. A comprehensive genetic map of the human genome based on 5,264 microsatellites. Nature 1996;380:152-4.
9 Slavotinek A, Rosenberg M, Knight S, et al. Screening for submicroscopic chromosome rearrangements in children with idiopathic mental retardation using microsatellite marker Genet 1999;36:405-11.

10 Knight SJL, Lese CM, Precht KS et al. An optimized set of human telomere clones for studying telomere integrity and architecture. Am f Hum Genet 2000;67:320-2.

11 Rosenberg M, Vaske D, Killoran CE, et al. Detection of chromosomal aberrations by a whole-genome microsatellite screen. Am $\mathcal{f}$ Hum Genet 2000;66:419-27. 